\title{
Effective Pair Potential of Liquid Ge and Sn Estimated from the X-Ray Structure Factor Data
}

\author{
K. Omote and Y. Waseda* \\ Kimura Metamelt Project, ERATO, Research Development Corporation of Japan \\ Yagiyama-minami 2-1-1-301, Sendai 982, Japan \\ *Institute for'Advanced Materials Processing, Tohoku University, Sendai 980-77, Japan
}

(Received May 16, 1995, final form July 1, 1995)

\begin{abstract}
Liquid polyvalent metals, such as $\mathrm{Ge}$ and $\mathrm{Sn}$, have a particular structural feature characterized by a small hump on the larger wavevector side of the first peak in their structure factors. Effective pair potentials of these elements have been obtained from the X-ray structure factor data using the modified hypernetted-chain (MHNC) equation. The effective pair potential of typical "normal" liquid metal, Na, was also estimated for comparison. The results suggest that the structural features of $\mathrm{Ge}$ and $\mathrm{Sn}$ are rather sensitive to the part of the pair potential around the second peak of the pair distribution function, whereas in the case of liquid $\mathrm{Na}$, its structural feature is almost determined by the part of the pair potential near the first peak region.
\end{abstract}

\section{INTRODUCTION}

The general profile of the structure factors $S(Q)$ of most liquid metals is known to be described by the hard-sphere model $/ 1 /$. On the other hand, the structure factors of some liquid polyvalent metals, such as $\mathrm{Ge}$ and $\mathrm{Sn}$, show a small hump on the larger wavevector side of the first peak in their structure factors. This particular feature could not be explained by such a simple hard sphere model.
Some theoretical approaches as to the structure of liquid polyvalent metals use effective pair potentials. For example, Mon, Ashcroft and Chester /2/ have suggested that the hump of the structure factor is attributed to the screened core-polarization effect. Nevertheless, the general profiles of liquid polyvalent metals are known to be described by the theoretical pair potentials derived from the simple empty-core pseudopotential $/ 3,4 /$. However, the theoretical pair potentials involve some physical assumptions which will in due course be explicitly examined. Further, it is still not clear which part of the pair potential plays an important role to give a particular small hump in the structure factor of liquid polyvalent metals.

With these facts in mind, the purpose of this paper is to estimate the effective pair potentials of liquid $\mathrm{Ge}$ and $\mathrm{Sn}$, using the Johnson-March scheme $/ 5$ / in a sense of the necessary condition for the best reproduction of the experimental structure factor data, including the lower wavevector region. For this purpose, we employ the modified hypernetted-chain (MHNC) equation $16 /$. The same approach has also been applied to liquid $\mathrm{Na}$ for comparison. In addition, we have also solved the MHNC equation again using the cut-off potential at the first dip of the pair distribution function in order to know the most effective part of the pair potential relevant to the hump of their structure factors. 


\section{METHOD}

When the interaction between particles in the liquid state is expressed as the sum of two-body forces, the pair distribution function $g(r)$ can be expressed by the following equation using the pair potential $u(r) / 7 /$

$$
g(r)=\exp [-\beta u(r)+g(r)-1-c(r)+B(r)]
$$

where $1 / \beta$ is the Boltzmann constant $k_{\mathrm{B}}$ times absolute temperature $T$. The direct correlation function $c(r)$ is expressed by the Ornstein-Zernike (OZ) relation,

$$
g(r)-1=c(r)+\int_{\nu}\left[g\left(r-r^{\prime}\right)-1\right] c\left(r^{\prime}\right) d r^{\prime}
$$

$B(r)$ is called a bridge function, which is the sum of all elementary graphs in cluster expansion. The set of eqs. (1) and (2) can be solved for a given potential $u(r)$, when the bridge function is known. Calculation for the contribution of all elementary graphs is generally impossible. The Percus-Yevick (PY) or the hypernetted-chain ( $\mathrm{HNC}$ ) approximation was frequently used $/ 7 /$ in the past to avoid such inconvenience by assuming the relation $B(r)=1+\operatorname{In} y(r)-y(r)$ or $B(r)=0$, where $y(r)=g(r) \cdot \exp [\beta u(r)]$. Rosenfeld and Ashcroft $/ 6 /$ have proposed substituting the bridge function of hard sphere potential, $B_{0}(r)$, for the exact bridge function of a given potential,

$$
g(r)=\exp \left[-\beta u(r)+g(r)-1-c(r)+B_{0}(r)\right] .
$$

This is called a modified hypernetted-chain (MHNC) equation and is known to yield a very accurate description of liquid structures with various potentials $/ 6,8,9 /$.

The hard sphere model for liquids is characterized by only one parameter of packing fraction, $\eta=\frac{\pi}{6} \rho \sigma^{3}$, where $\rho$ is the number density and $\sigma$ the diameter of the particles. Therefore, we must determine first the value of $\eta$ to obtain the appropriate bridge function of eq. (3). Lado, Foiles and Ashcroft $/ 10 /$ propose criteria for choosing the value of $\eta$ so as to minimize the free energy of a system.

$$
\int_{V}\left[g(r)-g_{0}(\eta ; r)\right] \frac{\partial B_{0}(\eta ; r)}{\partial \eta} d r=0,
$$

where $g_{0}(\eta ; r)$ is the pair distribution function of hard sphere liquids with $\eta$.

For hard sphere liquids, the exact solution of the PY equation is well known $/ 7 /$. However, the PY solutions are not necessarily in good agreement with the simulated results. With respect to this problem, Henderson and Grundke (HG) /11/ suggest a function $d(r)$ for the tail of the direct correlation function to obtain a more realistic hard sphere solution of the integral equation, as follows:

$$
c(r)=y(r)\{\exp [-\beta u(r)]-1\}+d(r) .
$$

It may be worth mentioning that this equation is identical to eq. (1) under the following condition,

$$
y(r)-1-\ln [y(r)]+B(r)=d(r) .
$$

In this work, the set of eqs. (5) and (2) was solved with the hard sphere potential using HG parametrization of $d(r)$ in order to obtain the pair distribution function $g_{0}(\eta ; r)$ and the bridge function $B_{0}(\eta ; r)$ for different packing fractions $\eta$. On the other hand, $g(r)$ and $c(r)$ are known to be obtained from the experimental structure factor data $S(Q)$ in the following equations,

$$
\begin{aligned}
& g(r)=1+\frac{1}{8 \pi^{2} \rho} \int[S(Q)-1] \exp (\mathrm{i} Q \cdot r) d Q, \\
& c(r)=\frac{1}{8 \pi^{3} \rho} \int \rho C(Q) \exp (\mathrm{i} Q \cdot r) d Q, \\
& \rho C(Q)=\frac{S(Q)-1}{S(Q)} .
\end{aligned}
$$

Then, the effective pair potential $u(r)$ can readily be obtained from

$$
\beta u(r)=g(r)-1-c(r)-\ln [g(r)]+B_{0}(\eta ; r),
$$

The value of the packing fraction $\eta$ could be determined by applying eq. (4) to the experimental $g(r)$ data.

It must be noted that some spurious ripples usually appear in the repulsive core part of the experimental $g(r)$ data calculated from $S(Q)$ using eq. (7a), due to the experimental uncertainties mainly arising from the fact that only the finite region of $S(Q)$ is available. For this reason, we assumed $g(r)=0$ in that region when using 
eqs. (4) and (8). The direct correlation function $c(r)$ was estimated directly from the experimental structure factor $S(Q)$. We did not modify $S(Q)$ to calculate $c(r)$ using eqs. (7b) and (7c), because the structure factor is a direct experimental result and it is not clear that the modification leads to more reliable data, especially in the low wavevector region. Then, the experimental $g(r)$ and $c(r)$ data do not satisfy the $\mathrm{OZ}$ relation of eq. (2) exactly, and the effect of this inconsistency will be discussed in the following section.

Fig. 1 shows the X-ray structure factor data $/ 12 /$ of three liquid metals, presently investigated. It is well known that the low wavevector region of the structure factor is very important for determination of the pair potential /13/. Therefore, these structure factor data in the low wavevector region were determined extensively down to $1 \mathrm{~nm}^{-1}$ by the transmission geometry /14/.

\section{EFFECTIVE PAIR POTENTIAL}

The effective pair potentials $\beta u(r)$ estimated from the X-ray structure factor data are shown in Figs. 2 to 4 together with each experimental pair distribution function $g(r)$. The values of the packing fraction $\eta$

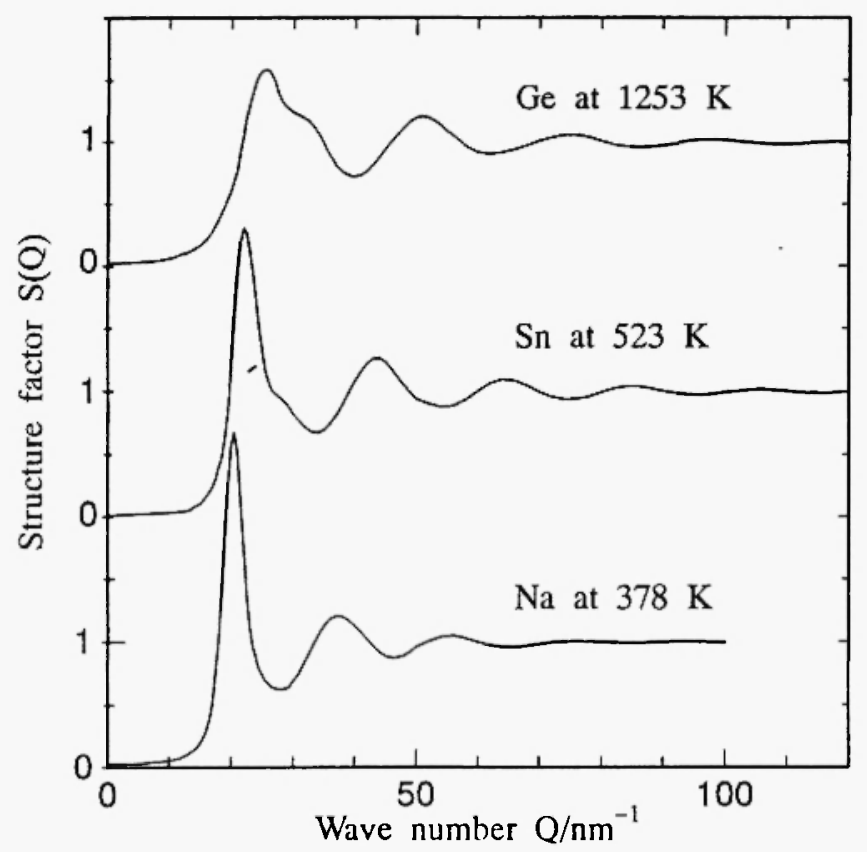

Fig. 1: $\mathrm{X}$-ray structure factors of liquid $\mathrm{Ge}, \mathrm{Sn}$ and $\mathrm{Na} / 12 /$.

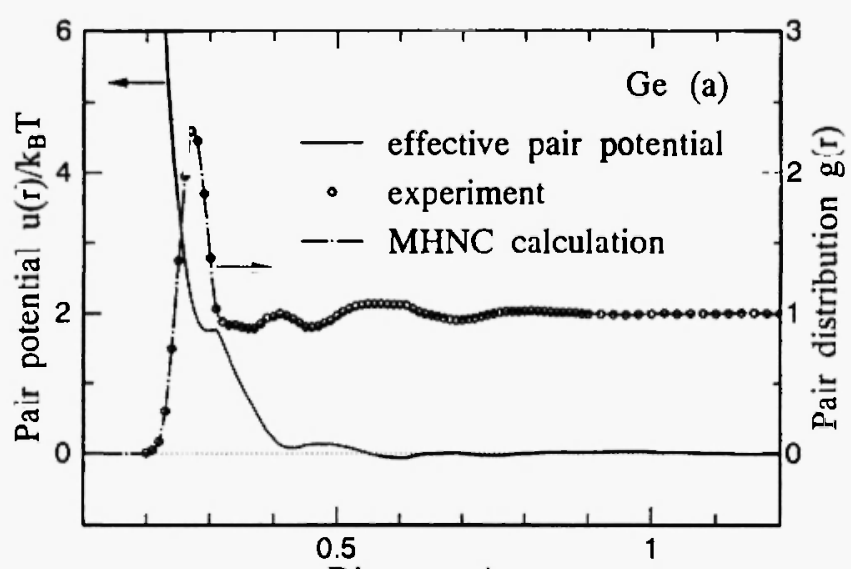

Distance $\mathrm{r} / \mathrm{nm}$

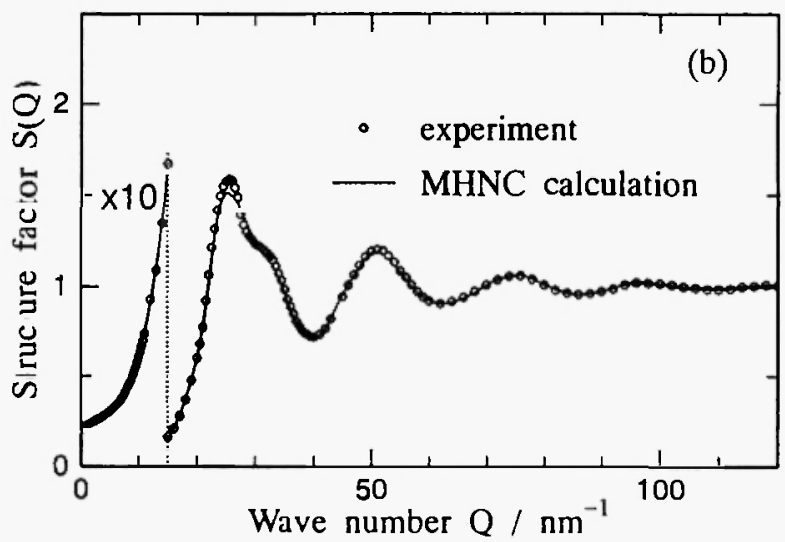

Fig. 2: (a) Effective pair potential of liquid $\mathrm{Ge}$ estimated from the $\mathrm{X}$-ray structure factor data and pair distribution function. (b) Structure factor.

given by eq. (4) are listed in Table 1 . In liquid $\mathrm{Na}$, the first peak of the pair distribution function appears to be located close to the minimum of the effective pair potential. In the case of liquid $\mathrm{Ge}$ and $\mathrm{Sn}$, the first peak of the pair distribution function is situated in the repulsive part of the effective pair potential. Such characteristic features are consistent with the results of these three liquid metals calculated by the pseudopotential theory $/ 3 /$.

To confirm the consistency of this work, we also estimated the structural functions of both $g(r)$ and $S(Q)$ from the obtained $\beta u(r)$ results. This was also based on the following reasons:

1) We assumed $g(r)=0$ in the repulsive core region when using eq. (8). this is well accepted because the 

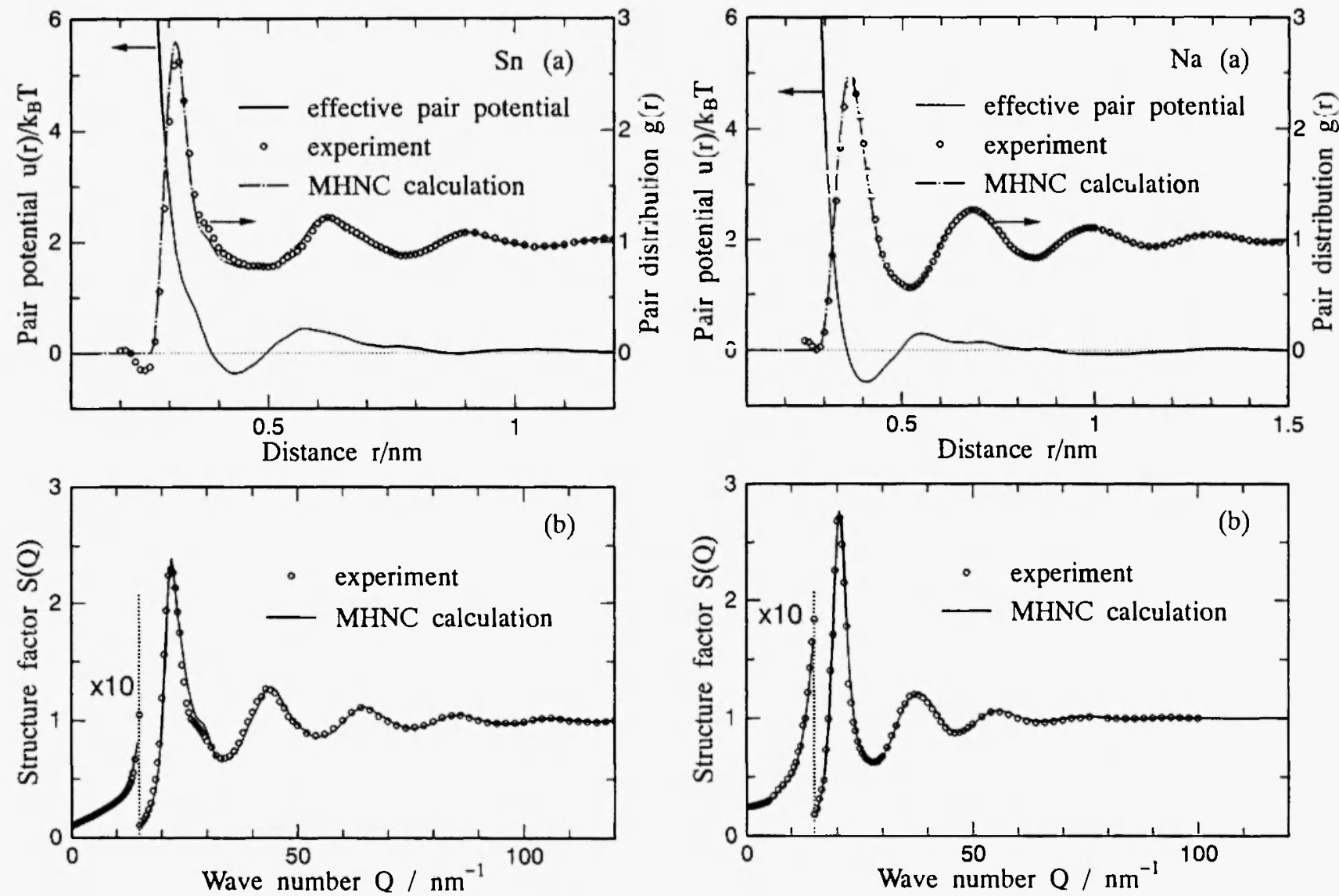

Fig. 3: (a) Effective pair potential of liquid Sn estimated from the $\mathrm{X}$-ray structure factor data and pair distribution function. (b) Structure factor.

Fig. 4: (a) Effective pair potential of liquid $\mathrm{Na}$ estimated from the X-ray structure factor data and pair distribution function. (b) Structure factor.

Table 1

Packing fraction of each liquid metal estimated by eq. (4) and atomic number density.

\begin{tabular}{c|ccc}
\hline Elements & $\mathrm{Ge}$ & $\mathrm{Sn}$ & $\mathrm{Na}$ \\
\hline $\begin{array}{c}\text { Temperature } \\
(\mathrm{K})\end{array}$ & 1253 & 523 & 378 \\
\hline $\begin{array}{c}\text { Density } \rho \\
\left(\mathrm{nm}^{3}\right)\end{array}$ & 46.1 & 35.2 & 24.3 \\
\hline $\begin{array}{c}\text { Packing } \\
\text { fraction } \eta\end{array}$ & 0.347 & 0.453 & 0.460 \\
\hline \hline
\end{tabular}


atoms do not mutually approach within the atomic core diameter due to the repulsion of the pair potential. However, the data set of $c(r)$ and such a modified $g(r)$ do not satisfy the $\mathrm{OZ}$ relation of eq. (2) in a strict sense and then the obtained potential $\beta u(r)$ might not be an exact solution of the MHNC equation.

2) We estimated $\beta u(r)$ from $S(Q)$ and $g(r)$ through eq. (8). However, it is also found that the resultant $S(Q)$ and $g(r)$ calculated with $\beta u(r)$ using the MHNC equation are often far from the input experimental data. This may be true in cases where the angular dependence of the interactions is important, and then the liquid structure may not be described by using two-body forces only.

The set of eqs. (2) and (3) was solved by iteration, and the integration in the $\mathrm{OZ}$ relation was carried out with a fast Fourier routine of 4096 points in the $Q$ space interval of $2 \pi / 40 \sigma$, where $\sigma$ is an approximate core diameter defined by $\sigma=(6 \eta / \pi \rho)^{1 / 3}$ The convergence is found to be fairly good by obtaining the solution within the uncertainty level of $10^{-6}$ in $300-800$ times iteration.

The resultant $S(Q)$ and $g(r)$ are shown in Figs. 2 to 4 together with the input experimental data. Good agreement is readily recognized not only for $\mathrm{Na}$, but also for $\mathrm{Ge}$ and $\mathrm{Sn}$. This strongly supports the conclusion that the present calculation for $\beta u(r)$ from the experimental data works well. It is reasonable to say that the structural profiles including a small hump of liquid $\mathrm{Ge}$ and $\mathrm{Sn}$ could be explained by using two-body forces only, although the three-body forces or higher order correlations are not completely excluded.

\section{ROLE OF THE LONG RANGE PART OF THE PAIR POTENTIAL}

The effective pair potentials of liquid $\mathrm{Ge}, \mathrm{Sn}$ and $\mathrm{Na}$ are now available and their usefulness has been demonstrated by reproducing the experimental structural functions of both $S(Q)$ and $g(r)$. Thus in this section, we will try to discover a possible origin of the small hump detected in the structure factors of $\mathrm{Ge}$ and $\mathrm{Sn}$ by artificially cutting the potential offiat the first dip of the corresponding pair distribution function and then calculating structural functions using the MHNC equation in order to investigate the effect of the longrange part of the pair potential.

The results are given in Figs. 5 to 7 . It may be stressed here that the cut-off effect of the long-range part of the pair potentials of liquid $\mathrm{Ge}$ and $\mathrm{Sn}$ induces the shift of the pair distribution functions as indicated by an arrow of $r_{2}$ in Fig. $6(0.58 \mathrm{~nm}$ for Ge and 0.62 $\mathrm{nm}$ for $\mathrm{Sn}$, respectively). This modification also clearly causes the disappearance of the small characteristic hump of the structure factor both for $\mathrm{Ge}$ and $\mathrm{Sn}$ as shown in Fig. 7. On the contrary, no significant variations are detected in liquid $\mathrm{Na}$ when applying a similar artificial modification to the pair potential. These results suggest that the long-range part of the pair potentials of liquid $\mathrm{Ge}$ and $\mathrm{Sn}$ plays a significant role in the particular small hump of their structure factors, whereas the structure of liquid $\mathrm{Na}$ is put mainly under the control of the short-range part of the pair potential.

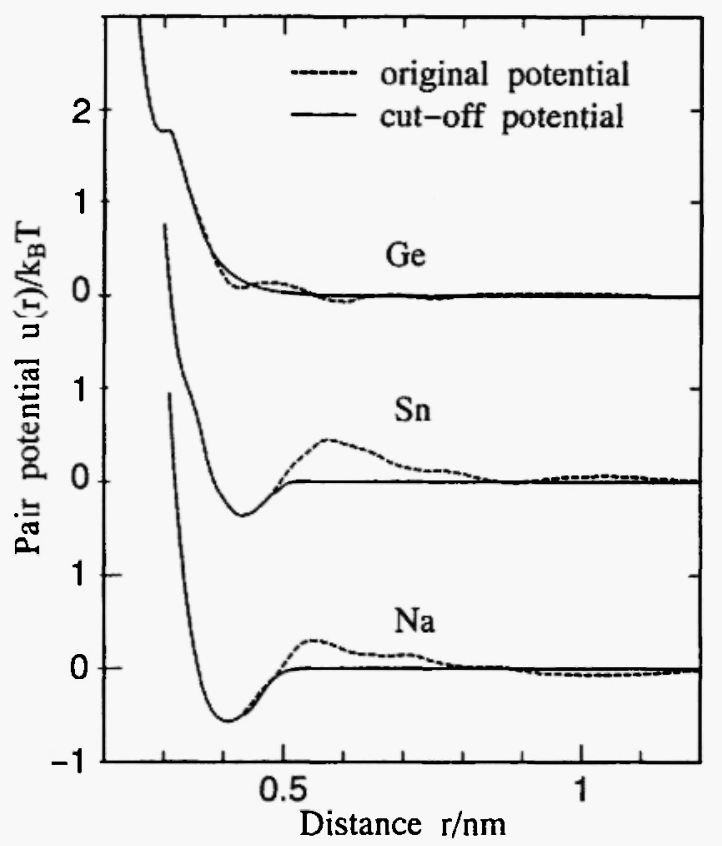

Fig. 5: Original and cut-off pair potentials of liquid $\mathrm{Ge}, \mathrm{Sn}$ and $\mathrm{Na}$. 


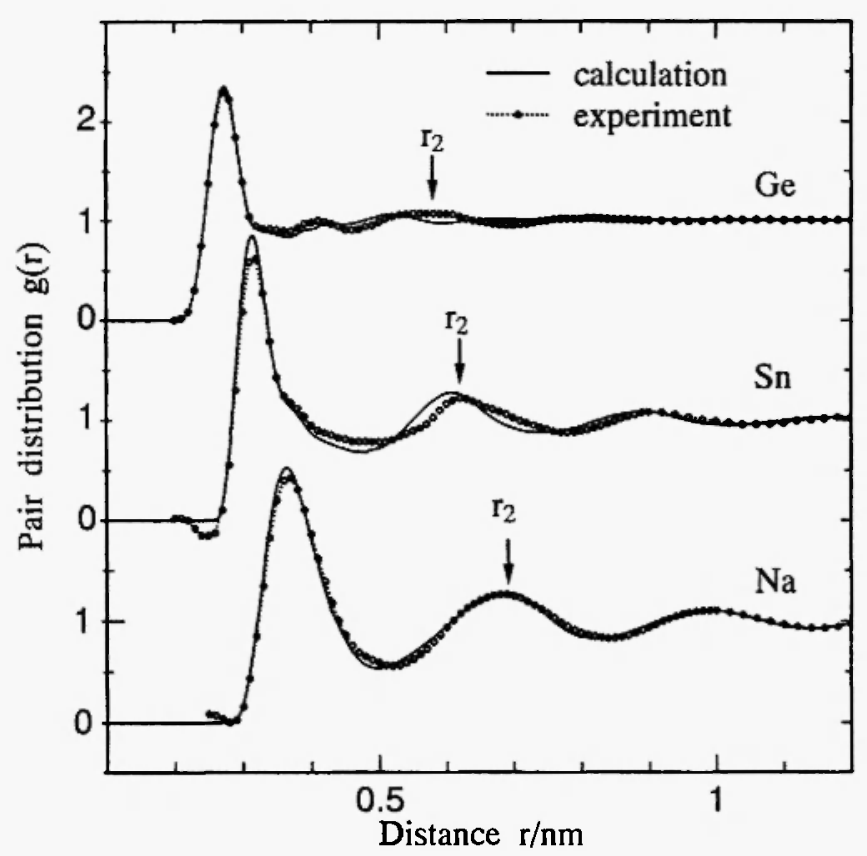

Fig. 6: Effect of the modification in the profile of the pair distribution functions of liquid $\mathrm{Ge}$, $\mathrm{Sn}$ and $\mathrm{Na}$.

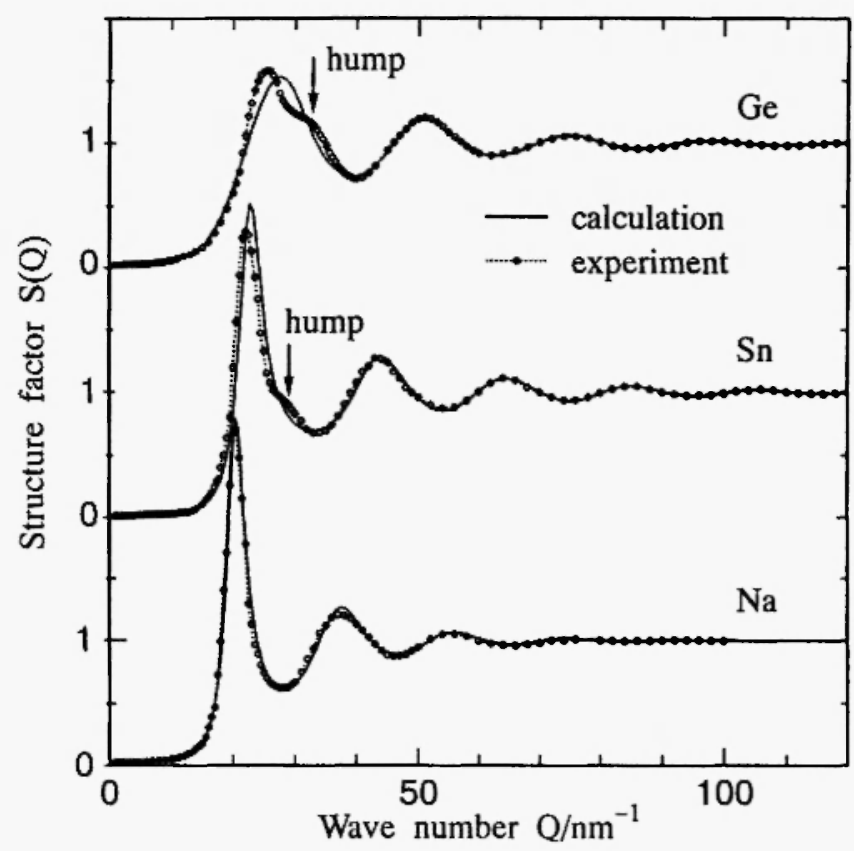

Fig. 7: Effect of the modification in the profile of the structure factors of liquid $\mathrm{Ge}, \mathrm{Sn}$ and $\mathrm{Na}$.

\section{CONCLUDING REMARKS}

The effective pair potentials of liquid $\mathrm{Ge}$ and $\mathrm{Sn}$ as well as $\mathrm{Na}$ have been estimated from the $\mathrm{X}$-ray structure factor data by using the MHNC equation. The obtained pair potentials are found to reproduce satisfactorily the experimental functions of $S(Q)$ and $g(r)$. The particular structural feature characterized by a small hump of the structure factor appears to be sensitive to the long-range part of the pair potential, as demonstrated in the Ge and Sn cases. Estimation of the effective pair potentials in liquid metals directly from the experimental structure factor data seems to be very encouraging, showing a significant step forward when using the MHNC equation. It would be interesting to extend this approach to other liquid metals, so that its usefulness and validity might be tested on a rather wider base.

\section{ACKNOWLEDGEMENTS}

The authors are greatly indebted to Dr. Kimura, the leader of the Kimura Metamelt Project, for his helpful support.

\section{REFERENCES}

1. N.W. Ashcroft and J. Lekner, Phys. Rev., 145, 83 (1966).

2. K.K. Mon, N.W. Ashcroft and G.V. Chester, Phys. Rev., B 19, 5103 (1979).

3. J. Hafner and V. Heine, J. Phys. F: Met. Phys., 13, 2479 (1983).

4. W. Jank and J. Hafner, Phys. Rev., B 41, 1497 (1990).

5. M.D. Johnson and M.H. March, Phys. Lett., 3, 313 (1963).

6. Y. Rosenfeld and N.W. Ashcroft, Phys. Rev., A 20, 1208 (1979).

7. J.-P. Hansen and I.R. McDonald, Theory of Simple Liquids, 2nd ed., Chap. 5, Academic Press, 1986.

8. G. Pastore and G. Kahl, J. Phys. F, 17, L267 (1987). 
9. D.J. Gonzalez, D.A. Ng and M. Silbert, J. NonCryst. Solids, 117 \& 118, 469 (1990).

10. F. Lado, S.M. Foiles and N.W. Ashcroft, Phys. Rev., A 28, 2374 (1983).

11. D. Henderson and E.W. Grundke, J. Chem. Phys., 63, 601 (1975).
12. Y. Waseda, The Structure of Non-Crystalline Materials, McGraw-Hill, 1980.

13. See for example: L.E. Ballentine and J.C. Jones, Can. J. Phys., 51, 1831 (1973).

14. Y. Waseda and S. Ueno, Sci. Rep. Res. Inst. Tohoku University, 34A, 15 (1988). 
